\title{
Unicornuate uterus with rudimentary horn, gynaecological and reproductive outcome
}

\author{
Arti Anand ${ }^{1}$, Madhuri Chandra ${ }^{2 *}$, Meeta Agarwal $^{3}$
}

\author{
${ }^{1}$ Department of Obstetrics and Gynecology, RKDF Medical College, Bhopal, Madhya Pradesh, India \\ ${ }^{2}$ Former Professor, Department of Obstetrics and Gynecology, Gandhi Medical College, Bhopal, Madhya Pradesh, \\ India \\ ${ }^{3}$ Consultant Obstetrics and Gynecology, Director Parul Hospital Bhopal, Madhya Pradesh, India
}

Received: 17 March 2021

Revised: 13 April 2021

Accepted: 14 April 2021

\section{*Correspondence:}

Dr. Madhuri Chandra,

E-mail: madhurichandra2@gmail.com

Copyright: ( $)$ the author(s), publisher and licensee Medip Academy. This is an open-access article distributed under the terms of the Creative Commons Attribution Non-Commercial License, which permits unrestricted non-commercial use, distribution, and reproduction in any medium, provided the original work is properly cited.

\begin{abstract}
Congenital malformations of the female genital tract occur in about 8-10\% of all women. The unicornuate uterus is a result of partial or complete agenesis of one of the paired Mullerian ducts. It could be a true unicornuate uterus or along with a rudimentary horn, which may be functional. When the rudimentary functional horn is noncommunicating, most patients become symptomatic after menarche or present with pregnancy related problems. Three clinical presentations of unicornuate uterus with non-communicating rudimentary horn encountered in last one year are described. The first case was of unruptured rudimentary horn pregnancy of 11-12 weeks. Second case had rudimentary horn removed as an adolescent due to intractable pain, but later main uterus harboured an uneventful pregnancy. Case three had two early abortions, followed by term caesarean section for fetal distress. With advent of ultrasonography and other imaging techniques, the diagnosis and management of unicornuate uterus and its variations is possible. The functional rudimentary horn if symptomatic must be removed along with ipsilateral tube. A unicornuate uterus is associated with obstetric problems and such women should be considered as high risk pregnancy.
\end{abstract}

Keywords: Unicornuate uterus with rudimentary horn, Adolescent and reproductive problems

\section{INTRODUCTION}

Congenital malformations of the female genital tract occur in about $8-10 \%$ of all women. ${ }^{1}$ The incidence differs depending on the population studied. The overall incidence is reported as $3.2 \%$ in women with normal reproductive outcomes, $5 \%$ to $10 \%$ in women with recurrent abortions in the first trimester, and greater than $25 \%$ in women with late first trimester and early secondtrimester pregnancy losses. ${ }^{2}$

The uterus, cervix and upper vagina develop from the two paired Mullerian (paramesonephric) ducts and developmental defects like failure of development of one or both of the Mullerian ducts or failure of fusion result in congenital malformations of female genital tract. The Mullerian and Wolffian ducts are closely linked embryologically and malformations of uterus and vagina are associated with anomalies of urinary tract, the kidneys and ureter. ${ }^{1}$

The unicornuate uterus is a result of partial or complete agenesis of one of the paired Mullerian ducts. It constitutes approximately $20 \%$ of all Mullerian duct anomalies, and most patients are asymptomatic until menarche or till they become pregnant. ${ }^{2}$

Two classifications of Mullerian duct anomalies exist, the most common is of American Society of Reproductive Medicine. ${ }^{1}$ The unicornuate uterus is under class 2 of 
ARSM, with sub classification by presence of rudimentary horn into a; communicating (10\%), b; noncommunicating (22\%), c; no cavity (33\%), and d; no horn $(25 \%)^{2}$

More recently the European Society of Human Reproduction and Embryology/European Society of Gastrointestinal Endoscopy (ESHRE/ESGE), have introduced a classification which terms the unicornuate uterus as hemi uterus and placed in class U4 with subclass a; with rudimentary (functional) cavity, may be communicating or not (U4a) and subclass b: without rudimentary cavity, horn without cavity or no horn (U4b).

\section{CASE SERIES}

Three clinical presentations of unicornuate uterus with non-communicating rudimentary horn encountered in last one year are described.

\section{Case 1}

RG, 21 years of age, married 1 year presented in emergency as amenorrhea 3 months, slight bleeding since morning and pain in abdomen. Her vitals were stable and internal examination revealed uterus normal size, slight blood stained discharge and cystic tender mass in left fornix. Per abdomen there was no tenderness or guarding.

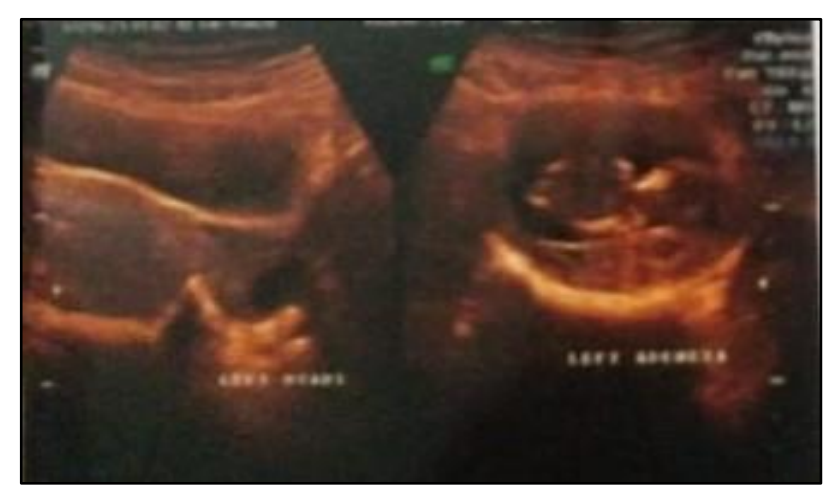

Figure 1: Case 1- Left rudimentary horn with ectopic pregnancy, USG reported as left adnexal ectopic pregnancy.

A left ectopic pregnancy was suspected and sonography revealed uterus normal in size and shape, endometrium hyperechoic. A gestational sac in left adnexa, fetus CRL $4.8 \mathrm{~cm}$ corresponding to 11 weeks 5 days, cardiac activity absent suggestive of left tubal ectopic. No free fluid in POD. (Figure 1)

She was taken for laparotomy, on opening abdomen, there was a unicornuate uterus with left rudimentary horn and a normal though short left fallopian tube (Figure 2). The left rudimentary horn was distended, cystic, thin walled with a flimsy attachment to the right sided uterus, but had not ruptured. It was excised along with the fallopian tube and content i.e. the ectopic pregnancy.
Histopathology report consistent with ectopic pregnancy. "One aspect of gestation sac related to smooth muscle bundles suggestive of uterine wall and other aspect wall of fallopian tube with focally preserved plicae".

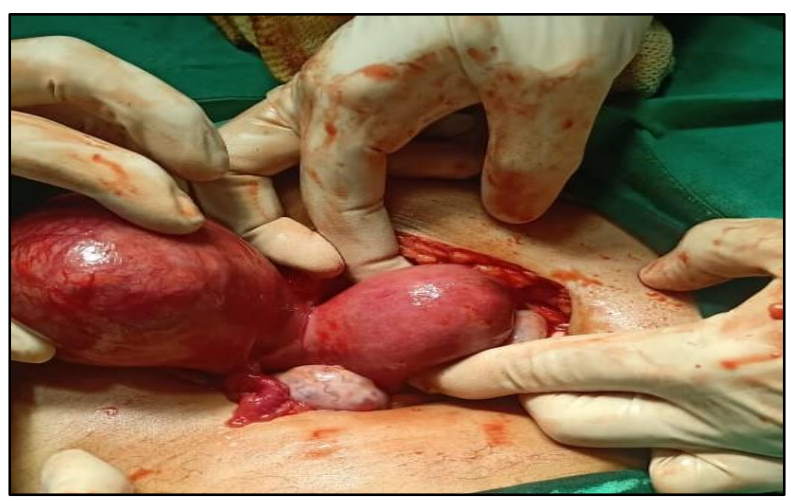

Figure 2: Case 1- Laparotomy picture, left rudimentary horn pregnancy. (Rudimentary horn distended with pregnancy).

Her post-operative period was uneventful and she was advised to go for ultrasonography to rule out any renal tract abnormalities.

\section{Case 2}

$\mathrm{ZB}$, was first seen by two of the authors about 16 years back when she presented at age 13 years with episodes of intractable pain during menstruation. She was prescribed analgesics and advised sonography, which did not reveal any uterine abnormality. She continued to visit the hospital over the next year with episodes of severe pain lasting throughout menstruation and a repeat USG showed a right unicornuate uterus with left rudimentary horn with hematometra, hematosalphinx and pelvic adhesions. There were no urinary tract abnormalities.

The findings were explained to child and mother and she was prescribed analgesics and injection DMPA monthly for 3 months. However there was no relief in pain and they were offered either drainage of hematometra under USG guidance or by laparoscopy. Both requested removal of the rudimentary horn and reassurances that her menstrual and reproductive function would not be affected by this surgery. A laparotomy with removal of left rudimentary horn and left fallopian tube was done. The rudimentary horn was placed quiet laterally, though it and the fallopian tube were distended with tarry blood, the attachment to right side was thin and small. The later end of fallopian tube was buried under adhesions along with left ovary which were separated and left ovary conserved. She came to OPD for a couple of follow up visits, when she reported to be pain free with minimal discomfort in menses.

ZB presented last year along with her well preserved previous records and discharge ticket requesting antenatal care, she was about 2 months pregnant at that time. Her 
antenatal period was smooth, she went into labour at 37 and half weeks and delivered a healthy baby boy.

\section{Case 3}

MS age 29 years, marital life 5 years, presented with history of 2 early spontaneous abortions. She was carrying a sonography which suggested right unicornuate uterus with left rudimentary horn, normal renal system. She was worried about her next pregnancy, though her menstrual cycle and other investigations (CBC, urine examination, RBS, thyroid profile, APLA and infection profile) were all normal.

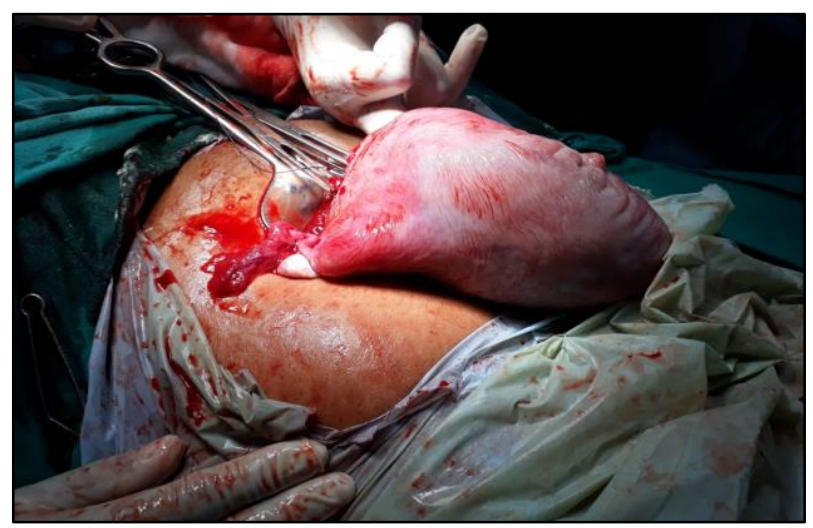

Figure 3: Case 3 - Fundal sacculation lodging placenta.

She conceived the next month and in view of her history was started on dydrogesterone twice daily. Scan at 6 weeks, showed fetus with presence of cardiac activity and normal chorionic tissue. USG for first trimester screening revealed normal fetus and a good cervical length of 4.3 $\mathrm{cm}$. Progesterone support was continued till 17 weeks (4 months), target scan detected no abnormality and cervical length was $4 \mathrm{~cm}$. Her pregnancy progressed well. At 39 weeks, she complained of decreased fetal movements. On examination uterus was towards right side, placed obliquely across abdomen, presentation cephalic and fetal heart sounds were normal. USG was ordered which revealed single live fetus of 39 weeks, placenta fundal grade 3 , and liquor adequate AFI $11 \mathrm{~cm}$, Biophysical Profile 10/10, colour flow normal (middle cerebral artery PSV 44.77, PI 1.32, umbilical artery PI 1.1, MCA PSV MOM 0.76 and cerebroplacental ratio 1.2). Sonologist had added a line, close monitoring with CTG advised.

In view of the high head, CPD an elective LSCS next morning was planned. Baby female delivered by head, cry immediate, there was fresh meconium stained liquor and delay in placental detachment. After delivery of placenta, uterus was exteriorised, the placenta was attached in a sacculation at the fundus, and uterine wall especially at fundus was comparatively thin (Figure 3). Post-operative period was uneventful with both mother and baby doing well.

\section{DISCUSSION}

We report 3 cases of unicornuate uterus with rudimentary horn, with different clinical presentation encountered last year. Incidentally all three were cases of unicornuate uterus with left side noncommunicating rudimentary horn, and having no urinary tract abnormalities. A unicornuate uterus causes few symptoms and is usually discovered by chance or as a result of pregnancy complications. ${ }^{1}$

A true unicornuate uterus or complete agenesis of one Mullerian duct is rare and is associated with absence of ipsilateral renal tract. Apparent unicornuate uterus with a rudimentary horn and fallopian tube on affected side is far more common (about 75\%). ${ }^{4}$ It occurs due to incomplete development of one Mullerian duct. Both kidneys are usually present but the one on affected side may be hypoplastic.

We searched PubMed for reports of pregnancy in the noncommunicating rudimentary horn with 104 results. Case 1 of patient RG was misdiagnosed on physical examination and sonography as left tubal ectopic. It was on laparotomy that true picture emerged, the rudimentary horn because of uterine musculature carries the pregnancy to a longer gestation than the fallopian tube. Here though the fetus was dead, the horn was distended but had not ruptured. Pregnancy in rudimentary horn is quite rare, incidence is reported as $1 / 75000$ to $1 / 150000$ pregnancies.4 The pregnancy in the non-communicating horn is probably caused by transperitoneal migration of sperm. ${ }^{2,4}$ Here it is then essential to remove the ipsilateral tube along with the pregnant rudimentary horn as it may be site of future ectopic pregnancy.

Case 2 presented to us first about 16 years back, we had done a laparotomy for excision of functional non communicating rudimentary horn as it was a cause of severe pain. In the present era, literature search suggests laparoscopic amputation of functional rudimentary horn. ${ }^{5}$ There are reports of better obstetric outcome if the two horns are united by reconstructive laparoscopic metroplasty to a single cavity. ${ }^{6,7}$

Pregnancy in unicornuate uterus is associated with poorer outcome in pregnancy. ${ }^{8}$ Obstetric problems are ectopic pregnancy, abortions, cervical incompetence, preterm labour, breech presentation and intrauterine fetal death. Growth restriction and fetal asphyxia may be due to the smaller uterine cavity, decreased muscle mass, abnormal uterine blood flow due to absence of blood flow contribution of contralateral ovarian artery.

\section{CONCLUSION}

The above three cases illustrate the different clinical presentations in unicornuate uterus with a noncommunicating rudimentary horn. With advent of ultrasonography and other imaging techniques, the 
diagnosis and management of unicornuate uterus and its variations is possible early with good reproductive outcomes. The functional rudimentary horn if symptomatic, either in form of hematometra or lodging an ectopic pregnancy, must be removed along with ipsilateral tube. A unicornuate uterus is associated with obstetric problems and such women should be managed as high-risk pregnancy.

Funding: No funding sources Conflict of interest: None declared

Ethical approval: Not required

\section{REFERENCES}

1. Malformations and maldevelopment of the genital tract. In: Narendra Malhotra, Jaideep Malhotra, Richa Saxena, Neharika Malhotra Bora editors. Jeffcoate's Principles of Gynecology. 9th edition. Jaypee brothers Medical Publishers. 2019;240-9.

2. Khati NJ, Frazier AA, Kathleen A, Brindle MD. The Unicornuate Uterus and Its Variants Clinical Presentation, Imaging Findings, and Associated Complications. Journal of Ultrasound in Medicine. 2012;31(2):319-31.

3. Grimbizis GF, Gordts S, Sardo ADS, Brucker S, De Angelis $\mathrm{C}$, Gergolet $\mathrm{M}$ et al. The ESHRE/ESGE consensus on the classification of female genital tract congenital anomalies. Human Reproduction. 2013;28(8):2032-44.
4. Sanchez-Ferrer ML, Prieto-Sanchez MT, del Campo FS. Variations in clinical presentation of unicornuate uterus with non-communicating rudimentary horn (class IIB of the American Fertility Society classification) Taiwanese J Obstet Gynecol. 2018;57:110-4.

5. Yen CF, Pai A, Lo A, Chueh HY, Huang HY, Lin SL. Laparoscopic metroplasty: reconstructive surgery for unicornuate uterus with noncommunicating, functional uterine horn. Fertil Steril. 2020;114(5):1119-21.

6. Mabrouk M, Arena A, Zanello M, Raimondo D, Seracchioli R. Unicornuate uterus with noncommunicating functional horn: diagnostic workup and laparoscopic horn amputation. Fertil Steril. 2020;113(4):885-7.

7. Zhang Y, Liu S, Zhang X, Ding Y, Hua K. Laparoscopic metroplasty for unicornuate uterus with a functional noncommunicating rudimentary horn. Int J Gynaecol Obstet. 2020;149(1):31-6.

8. Reichman D, Laufer MR, Robinson BK. Pregnancy outcomes in unicornuate uteri: a review. Fertil Steril. 2009;91(5):1886-94.

Cite this article as: Anand A, Chandra M, Agarwal M. Unicornuate uterus with rudimentary horn, gynaecological and reproductive outcome. Int J Reprod Contracept Obstet Gynecol 2021;10:2055-8. 